\title{
Social cognitive factors associated with physical activity among university students: a cross-sectional study
}

\begin{abstract}
The transition from secondary school to university is often accompanied by unhealthy behaviour pattern of decreasing physical activity. The role of social cognitive factors affecting physical activity among university students is not well explored to date. This study was aimed to determine the influence of Self-efficacy, Outcome Expectation for Exercise and Social Support for Exercise Behaviour on physical activity participation among university students. A cross-sectional survey was conducted among university students in Malaysia using a self-reported questionnaire. A total of 390 students participated in the survey. A comprehensive questionnaire was used to collect data from participants on socio-demographic, psychosocial cognitive characteristics and their level of physical activity. Our findings revealed that $63.8 \%$ of participants belong to normal range of body mass index while underweight, overweight and obese are 20.3, 12.3 and $3.6 \%$ respectively. $75.89 \%$ of participants did not meet the recommended vigorous intensity exercise per week. $92.82 \%$ of students participated in less than 5 days of moderate physical activity. Self-efficacy and outcome expectations for exercise had the strongest relations to physical activity; self-efficacy remained the strongest predictor of physical activity. Social support for exercise from family was found to have a weak correlation to physical activity level compared to other variables. Social cognitive factors exert independent effects on physical activity within this population. The findings of this study may facilitate the development of effective and tailored multilevel intervention programs paying more attention to social cognitive factors that will in turn increase physical activity among university students.
\end{abstract}

Keywords: physical activity, self-efficacy, social support, university students, outcome expectation, Malaysia
Volume 2 Issue 2 - 2017

\author{
Majeed NA,' Jabbar MA, ${ }^{2}$ Jun $X C^{\prime}$ \\ 'Department of Physiotherapy, University Tunku Abdul Rahman, \\ Malaysia \\ ${ }^{2}$ Department of Population Medicine, University Tunku Abdul \\ Rahman, Malaysia
}

Correspondence: Nizar Abdul Majeed Kutty, Department of Physiotherapy, University Tunku Abdul Rahman, Malaysia,Tel 0060163702 375, Email nizarkualalumpur@gmail.com

Received: April 30, 2017| Published: May 23, 2017

\section{Introduction}

Research on physical activity (PA) participation has broadened beyond a focus on the effect of intrapersonal factors alone, towards the application of the social cognitive model of health to physical activity. This new focus has revealed that interpersonal, environmental and policy related factors influence PA participation. ${ }^{1}$ However, there has been limited contextualization of these multiple and interacting elements for specific population groups. Young adulthood is when PA patterns are established; such patterns are likely to be maintained throughout adulthood and therefore affect an individual's lifelong health. ${ }^{2}$ In particular, college is an opportune time for self-directed learning and modification of lifestyle behaviors. During adolescences and in the transition to university and specifically during the duration of study at university, the disregard to healthy lifestyle is on the increase and the decrease in the practice of moderate to vigorous PA is prevalent. There is evidence that a larger proportion of students transiting to university engage in low levels of PA, with about onethird of previously active students becoming inactive during the transition. ${ }^{3}$ In addition, an increased risk of secondary disabilities from psychological factor is most evident in adolescence. ${ }^{4}$

According to a report from The British Scientific Journal Lancet in July 2012, Malaysia is in the list of the top ten most physically inactive countries in the region with about $61.4 \%$ of Malaysians aged 15 and above considered physically inactive. ${ }^{5}$ Physical inactivity levels are rising in developing countries and Malaysia is of no exception. ${ }^{6} \mathrm{~A}$ good body of studies state that demographics (e.g. age, gender), psychological factors (e.g. self-efficacy, perceived enjoyment), social factors (social support from family and friends) could be possible influencing factors of university students' PA behavior. ${ }^{7,8}$ According to World Health Organization - Non Communicable Diseases country profiles-2011, globally the prevalence of physical inactivity is on the rise in many countries including Malaysia. Based on the 2008 estimated prevalence, it showed that about $60.5 \%$ of adult Malaysians aged 15 years and above are physically inactive, with male $56.0 \%$ and females $65.0 \%$, thus enlisting Malaysia among the top ten physically inactive nations in Asian region. ${ }^{9}$ In 2014 meeting of the World leaders to assess the effort made so far in combating NCDs from 2011, the 2014 NCD country profiles showed not enough progress across all the countries. A target of $10 \%$ reduction in prevalence of insufficient PA is among the 9 targets set for year $2025 .{ }^{10}$ The university environment is a setting with much greater advantages and easier opportunities towards improving PA among students. However in university, unlike in primary and secondary schools, the absence of proper structure on physical education, health promotion and, making the students most often to neglect the opportunities of utilizing the available facilities effectively. Findings may also help update records in undergraduate prospectus as in relates to PA. It can also be used to reflect prevalence of age group 18-25 in the general population since university is a composition of the general public in terms of race and gender.

A growing body of literature points out that higher PA levels are associated with lower health risks including overweight and obesity related diseases. ${ }^{11,12}$ Regular PA remains an important behavior for promoting health, postponing or preventing prevalent musculoskeletal 
disorders such as mechanical low back pain, neck and shoulder pain and decreasing the risk of developing coronary heart disease, hypertension, diabetes, osteoporosis, obesity and colon cancers. ${ }^{13}$ Understanding why young people do not engage in PA is important for intervention efforts encouraging more active lifestyles. One model commonly used in predicting and explaining health behavior that can be used to examine adolescent PA with this population is Social Cognitive Theory (SCT). SCT is a robust theoretical framework for explaining and predicting PA. SCT basically uses personal, psychosocial and environmental factors to predict behavior. Key SCT factors of PA are self-efficacy, social support, goal setting, outcome expectation, and the environment. Self-efficacy is a key to regular exercise, while environmental factors including social support and the environment are important for exercise compliance. In addition, outcome expectation affects motivation to participate in specific activities and is thus important for college students' PA. ${ }^{14}$

It is important to develop an understanding of the influences on physical activity among youth to identify potential mediators of behavior ${ }^{15}$ and develop effective interventions. ${ }^{16}$ Understanding the influences on PA behavior change is difficult because they change over the life course. ${ }^{17}$ While these influences are often referred to as determinants of PA, most studies conducted in Malaysia are not conclusive. First generation students transitioning to college experience specific challenges that impact on their engagement in physical activity. Furthermore, this population experiences a context disruption that provides a unique opportunity to examine whether social cognitive factors predict physical activity. Most prior studies on college students' PA conducted in other countries focused on demographic factors such as age, body mass index (BMI), perceived health and sitting behavior. ${ }^{18,19}$ Though many studies had been conducted to determine the level of PA among university students the role of social cognitive factors is not well explored. While PA is valued across different segments of many cultures, given the several culture-specific predictors, physical activity appears to be more transversal than universal. ${ }^{20}$ More research on social cognitive factors among university students is still warranted to better understand energy expenditure behaviors in order to develop effective strategies aiming to increase physical activity. Therefore, the aim of this study was to investigate the association of social cognitive factors and PA among a sample of university students from Malaysia.

\section{Materials and methods}

We adopted a cross-sectional study design to determine the association of social cognitive factors and PA among university students. Participants were recruited from Sungai Long campus of University Tunku Abdul Rahman, Selangor, Malaysia. A total of 390 students participated in the survey. Subjects of this study had to be a Malaysian citizen enrolled as a full-time student aged in between 18 to 25 years old that can understand English. Students who had physical limitations hindering physical activity such as pregnancy, neurological disorder, cardiopulmonary disease, musculoskeletal problems and blindness were excluded from this study. The researchers obtained ethical approval from Scientific and Ethical Review Committee of University Tunku Abdul Rahman, Malaysia. Informed consent was obtained from all participants and data were encrypted to ensure confidentiality. We conducted a questionnaire survey to collect data from participants. The questionnaire consisting of five sections took approximately 20 minutes to complete. In the first section, basic demographic details about the participants were elicited. Information regarding socio-demographic variables i.e. age, education, gender, marital status, living arrangement and self-reported body weight and height were sought from the participants. Body mass index (BMI) was computed from self-reported height and weight $\left(\mathrm{kg} / \mathrm{m}^{2}\right)$. It was grouped into four categories: underweight $(<18.5)$, normal weight (18.5-24.9), overweight (25.0-29.9) and obese $(\geq 30.0)$. Second section was aimed to determine the physical activity level of participants. The remaining sections of the questionnaire were targeted to determine the Self-efficacy, Outcome Expectation for Exercise and Social Support for Exercise Behavior.

\section{Outcome measurements}

\section{International physical activity questionnaire (IPAQ- SF)}

To measure physical activity, we used an English version of a short self-administered International Physical Activity Questionnaire (IPAQ). This version of IPAQ is meant for use among young and middle-aged adults and has an acceptable test-retest reliability and criterion validity. ${ }^{21}$ IPAQ measures the frequency and average duration of vigorous-intensity, moderate-intensity and walking activities in the past 7 days. The total duration of PA was classified as "sufficient" or "insufficient" according to the PA guidelines for health benefits. Sufficient PA for health benefits for adults is defined as moderateintensity aerobic PA for a minimum of 30 minutes on 5 days/week or vigorous-intensity aerobic activity for a minimum of 20 minutes on 3days/week, while insufficient PA is defined as not meeting the sufficient PA standards. Intra-class correlation coefficients (ICCs) between day-1 and day-9 assessments for IPAQ total and vigorous PA were high in young adults $(0.84$ to 0.93$) .^{22}$

\section{Exercise self-efficacy scale (ESEs)}

Self-efficacy of participants in exercise was measured using Exercise Self-efficacy scale (ESEs). It consisted of 10 questions and each question has 4 options. Participants had to answer all 10 questions. This scale assesses the confidence in participant's ability to maintain exercise under all circumstances on a 4-point scale from 1 (not confident at all) to 4 (very confident). The total score is calculated by finding the sum of 10 items. Scores range from 10-40. A higher score indicates more exercise self-efficacy. There is sufficient evidence of internal consistency (alpha $=0.92$ ), and a squared multiple correlation coefficient using structural equation modelling provided further evidence of reliability ( $\mathrm{R} 2$ ranged from 0.38 to 0.76 ). ${ }^{23}$

\section{Multidimensional outcome expectations for exercise scale (MOEES)}

The Multidimensional Outcome Expectations for Exercise Scale was used to assess three related, but conceptually independent domains of outcome expectations for exercise (i.e., physical, social and self-evaluative outcome expectations). For each item, participants indicate the degree to which they agree with each statement (e.g., "Exercise will increase my muscle strength") on a scale of 1 (strongly disagree) to 5 (strongly agree). Its internal consistency ranges from $0.72-0.93$ and Cronbach's $\alpha$ is reported as 0.87 .

\section{Social support for exercise behavior scale (SSEBs)}

This scale was used to determine the social support or influence by family and friends toward exercise in participants. It consisted of 13 questions and each question has two components which are 
Table 5 Correlation between moderate exercise in last 7 days and exercise self-efficacy

Correlation Between Moderate Exercise in Last 7 Days and Exercise Self-Efficacy

Moderate Exercise in last 7 days

Pearson Correlation

1

Sig. (2-tailed)

$\mathrm{N}$

Pearson Correlation

Exercise Self Efficacy Score

Moderate Exercise in last 7 days
Exercise Self Efficacy Score
Sig. (2-tailed)

$\mathrm{N}$
*Correlation is significant at the 0.05 level (2-tailed).

\section{Discussion}

The identification of physical activity correlates can help to develop effective PA interventions. In the current study, a number of demographic and social cognitive variables were examined in an attempt to understand their relationship with participation in PA among a sample of university students from Malaysia. The current study identified that majority of the participants have normal BMI. Our findings are in accord with a similar study conducted among university students in Malaysia that reported healthy weight had the highest frequency for BMI which accounted $68.1 \% .{ }^{25}$ BMI could be lower due to decreased energy intake (food), due to increases in energy expenditure or both. Perhaps most importantly and unlike weight or BMI, fitness is very sensitive to changes in physical activity behavior. Fitness is the single most important indicator of someone's health and can be measured safely and objectively in the general population. According to a recent study from University of Essex; continued reliance on BMI as the lone measurement of child health is not working. ${ }^{26} \mathrm{BMI}$ models are incomplete, leaving shorter individuals to believe they are healthy and taller people feel they have an issue with their weight. Although BMI does not directly measure body fat, its utility as a risk estimate has been demonstrated in multiple population studies. ${ }^{27}$ Nonetheless, in some instances, the use of height and weight alone for calculation of BMI as a surrogate measure of body fat may lead to an incorrect estimation of risk. In fact, BMI does not accurately reflect the presence or severity of the health risk. ${ }^{28}$

PA level of participants in this study was found quite low. The findings of a study among university students in Malaysia revealed that the prevalence of physical inactivity among the respondents was $41.4 \% .^{29}$ The current study's reliance on self-reported data is a limitation. However, the students involved in the study were young adults who are capable of recalling physical activity behavior at a satisfactory level. Additionally objective measures are quite expensive. Evidence from Malaysia also suggests ethnic differences in physical activity levels. A study by Teh $\mathrm{CH}$ found "Other" (usually indigenous or mixed) races to be most active physically, and Chinese the least active, with Malay and Indian participants falling in between. ${ }^{30} \mathrm{~A}$ big majority of the participants of this study were of Chinese descent. The finding of this study on levels of PA among university students is alarming. Generally, physical activity tends to decrease with age. Hawkins found this to be true for all ethnicities and races in the USA. ${ }^{31}$ A study of 19,145 Malaysians produced similar results. Specifically, university students who have greater levels of education and those that presumably have more discretionary time seem to be choosing not to be physically active. This points to a fundamental attitude among many Malaysians, especially ethnic Chinese and those with more education; that physical activity is not a priority or not desirable.

The findings of our study revealed that there is a positive association between self-efficacy and levels of PA $p<0.05$, the prevalence of physical inactivity is significantly higher among those with low selfefficacy compared with those with high self-efficacy. Self-efficacy has received the most consistent support of any psychosocial factor as a strong determinant of physical activity behavior. Further, our cross-sectional findings should be interpreted with caution as it is also possible that increased PA resulted in greater self-efficacy an idea inherent in the reciprocal nature of SCT ${ }^{32}$ However, other researchers have found that self-efficacy is a consistent predictor of PA behavior and the findings from this study show similar strong support for selfefficacy. ${ }^{33}$

Additionally, statistically significant associations were found between outcome expectations of exercise and PA. These findings indicate that those students who recognize the benefits of activity and identify fewer barriers are more active than those students who do not. Despite receiving less attention than self-efficacy in the literature, there is strong support for the relationship between outcome expectations and physical activity levels activity. ${ }^{34}$ In previous epidemiological research, it has been found to have inconsistent associations with physical activity. ${ }^{35}$ Outcome expectancies are beliefs about the likelihood that PA will produce benefits or undesired outcomes and can include social or appearance related beliefs. If an individual believes a behavior is linked to a desirable outcome, there is a greater likelihood of regular PA. ${ }^{36}$ Outcome expectancy has played a crucial role in the development of cognitive explanations of behavior. For example, outcome expectancy has been theorized to explain the association inherent in stimulus response theory and as the mechanism of classical and operant conditioning. Today's health behavior models fit within this larger social cognitive framework, in which outcome expectancy remains an integral part. New directions in outcome expectancy research could involve expanding the conceptualization of outcome expectancy to include expected outcomes of sedentary behavior and affective responses to physical activity.

Small but significant associations between all available providers of total social support and physical activity were found. The findings indicate the types of general social support that facilitate or hinder participation in PA. Our findings reflect that peer support is more significant than family support in predicting PA. Majority of the 
participants were living away from parents. So the influence of friends was found to be more than family support in influencing physical activity participation. Sallis et al. ${ }^{37}$ found that the most consistent influence on physical activity participation was peer support. ${ }^{37}$ Furthermore, acceptance by peers, perceptions of and interactions with peers become increasingly important as adolescents further define their identity apart from membership in their family. A recent meta-analysis suggests that social support is not a strong predictor of PA in adolescent girls though parents and friends may have a role in enhancing PA. ${ }^{38}$ A systematic review on the effectiveness of interventions to increase PA also showed social support is of limited utility as a sole intervention. ${ }^{39}$ The findings of this study indicate that socio-environmental factors in addition to personal factors may increase the likelihood of participation in PA among university students. Intervention programs taking the target group's opinions into account may be more likely to succeed in the long run.

\section{Limitations and recommendations}

There were several limitations for the present study. First, the data were measured by self-report questionnaire that included different measurement scales including three and four-response options. It is possible that small sets of response options lower the absolute magnitude of variability and therefore create a statistical artifact in correlation analyses. Another limitation is the cross-sectional nature of the study which makes it impossible to conclude about antecedents of successful exercise behavior change. Adolescents may make behavioral choices during this developmental period that contribute to lifelong behavioral patterns; however, longitudinal studies are needed to research this area. Further research is necessary to clarify the role that siblings may play in PA behavior in young adults.

\section{Conclusion}

In the current study, self-efficacy and outcome expectancy of exercise were key predictors of participation in PA. Adolescents need to develop the necessary skills and knowledge to overcome potential barriers to an active lifestyle. University life is a critical period regarding unhealthy changes in energy related behaviors in students. Universities can help influence them to make healthy choices by providing them with different programs and choices such as but not limited to: lifestyle management, self-management strategies, enrichment healthy routine programs, focus groups, and psychoeducational awareness sessions for both adolescents and other family members and caregivers. Future development of interventions should take account of the socially interactive nature of PA.

\section{Acknowledgements}

None.

\section{Conflict of interest}

The author declares no conflict of interest.

\section{References}

1. Sallis J, Cervero RB, Ascher W, et al. An ecological approach to creating active living communities. Annu Rev Public Health. 2006;27:297-322.

2. Choi JY, Chang AK, Choi EJ. Sex differences in social cognitive factors and physical activity in Korean college students. J Phys Ther Sci. 2015;27(6):1659-1664.
3. Sinclair KM, Hamlin MJ, Steel G. Physical activity levels of first-year New Zealand university students: a pilot study. Youth Studies Australia. 2005;24(1):38.

4. Favlo D. Medical and psychosocial aspects of chronic illness and disability. USA: Jones and Bartlett Publishers; 2009. 693 p.

5. Global recommendations on physical activity for health. Switzerland; World health organization; 2010.

6. Guthold R, Ono T, Strong KL, et al. Worldwide variability in physical inactivity a 51-country survey. Am J Prev Med. 2008;34(6):486-494.

7. Haase A, Steptoe A, Sallis JF, et al. Leisure-time physical activity in university students from 23 countries: associations with health beliefs, risk awareness and national economic development. Prev Med. 2004;39(1):182-190.

8. Simons D, Clarys P, Bourdeaudhuij DI, et al. Why do young adults choose different transport modes? A focus group study. Transport Policy. 2014;36:151-159.

9. NCD Country profiles. Switzerland: World health organization; 2011.

10. NCD Country profiles. Switzerland: World health organization; 2014.

11. Warburton DE, Nicol CW, Bredin SS. Health benefits of physical activity: the evidence. CMAJ. 2006;174(6):801-809.

12. Jakicic JM, Davis KK. Obesity and physical activity. Psychiatr Clin North Am. 2011;34(4):829-840.

13. Jones DA, Ainsworth BE, Croft JB, et al. Moderate leisure-time activity: who is meeting the public health recommendations? A national cross sectional study. Arch Fam Med. 1998;7(3):285-289.

14. Rovniak LS, Anderson ES, Winett RA, et al. Social cognitive determinants of physical activity in young adults: a prospective structural equation analysis. Ann Behav Med. 2002;24(2):149-156.

15. Baranowski T, Jago R. Understanding the mechanisms of change in children's physical activity programs. Exerc Sport Sci Rev. 2005;33(4):163168 .

16. Bungum T, Dowda M, Weston A, et al. Correlates of physical activity in male and female youth. Pediatric Exercise Science. 2000;12(1):71-79.

17. Sallis JF, Hovell MF. Determinants of exercise behavior. Exerc Sport Sci Rev. 1990;18:307-330.

18. Dowda M, Ainsworth BE, Addy CL, et al. Correlates of physical activity among U.S. young adults, 18 to 30 years of age, from NHANES III. Ann Behav Med. 2003;26(1):15-23.

19. You JS, Chin JH, Kim MJ, et al. College students' dietary behavior, healthrelated lifestyles and nutrient intake status by physical activity levels using international physical activity questionnaire (IPAQ) in Incheon area. Korean Journal of Nutrition. 2008;41:818-831.

20. Seo DC, Torabi MR, Jiang N, et al. Cross-cultural comparison of lack of regular physical activity among college students: universal versus transversal. Int J Behav Med. 2009;16(4):355-359.

21. Craig CL, Marshall AL, Sjöström M, et al. International physical activity questionnaire: 12-country reliability and validity. Med Sci Sports Exerc. 2003;35(8):1381-1395.

22. Papathanasiou G, Georgoudis G, Papandreou M, et al. Reliability measures of the short international physical activity questionnaire (IPAQ) in Greek young adults. Hellenic J Cardiol. 2009;50(4):283-294.

23. Resnick B, Jenkins LS. Testing the reliability and validity of the selfefficacy for exercise scale. Nurs Res. 2000;49(3):154-159. 
24. Reis MS, Reis RS, Hallal PC. Validity and reliability of a physical activity social support assessment scale. Rev Saude Publica. 2011;45(2):294-301.

25. Kutty NAM, Aziz AA. Association of Sedentary Behaviour and Cardiometabolic Risk Biomarkers among Chinese Females: A Crosssectional Study. Journal of Obesity \& Weight Loss Therapy. 2016;6:304.

26. Sandercock G. Poor fitness is a bigger threat to child health than obesity. London: The Conversation; 2017.

27. Prospective Studies Collaboration, Whitlock G, Lewington S, Sherliker $\mathrm{P}$, et al. Body-mass index and cause-specific mortality in 900,000 adults: collaborative analysis of 57 prospective studies. Lancet. 2009;28(373):1083-1096.

28. Kushner RF. Clinical Assessment and Management of Adult Obesity. Circulation. 2012;126(24):2870-2877.

29. Goje M, Salmiah MS, Azuhairi A, et al. Physical inactivity and its associated factors among University Students. Journal of Dental and Medical Sciences. 2014;13(10):119-130.

30. Teh CH, Lim KK, Chan YY, et al. The prevalence of physical activity and its associated factors among Malaysian adults: Findings from the National Health and Morbidity Survey 2011. Public Health. 2014;128(5):416-423.

31. Hawkins MS, Storti KL, Richardson CR, et al. Objectively measured physical activity of USA adults by sex, age and racial/ethnic groups: A cross-sectional study. Int J Behav Nutr Phys Act. 2009;6:31.

32. Bandura A. The Exercise of Control. Self-Efficacy, USA: WH Freeman; 1997. $604 \mathrm{p}$

33. Auley ME, Blissmer B. Self-efficacy determinants and consequences of physical activity. Exerc Sport Sci Rev. 2000;28(2):85-88.

34. Pinto BM, Lynn H, Marcus BH, et al. Physician-based activity counseling: Intervention effects on mediators of motivational readiness for physical activity. Ann Behav Med. 2001;23(1):2-10.

35. Sallis JF, Prochaska JJ, Taylor WC. A review of correlates of physical activity of children and adolescents. Med Sci Sports Exerc. 2000;32(5):963975.

36. Bandura A. Health promotion by social cognitive means. Health Educ Behav. 2004;31(2):143-164.

37. Sallis JF, Taylor WC, Dowda M, et al. Correlates of vigorous physical activity for children in grades 1 through 12: Comparing parent-reported and objectively measured physical activity. Pediatric Exercise Science. 2002;14(1):30-44.

38. Laird Y, Fawkner S, Kelly P, et al. The role of social support on physical activity behaviour in adolescent girls: a systematic review and metaanalysis. Int J Behav Nutr Phys Act. 2016;13:79.

39. Kahn EB, Ramsey LT, Brownson RC, et al. The effectiveness of interventions to increase physical activity: A systematic review. Am J Prev Med. 2002;22(4):73-107. 